\title{
An Implementation of Adaptive Classification Exploration for Channel Estimation in Mu-Mimo
}

\author{
B. Adhithya, S. Vinay kumar, S. Mahesh Reddy
}

\begin{abstract}
A gigantic multi-customer versatile multi-input different yield (MIMO-MU) Wireless Communications structures, reference to the base station $(B S)$ is made is furnished with a tremendous In LTE faster (LTE-A) without modulation position pictures are utilized for pilot upheld station estimation to perform clear disclosure. Since these reference pictures are conveyed at the vague time-repeat places for whole customers of Multiuser MIMO (MIM-MU) movements of the each and every spatial layer in Solo-User MIMO (MIMO-SU) proposed to be code-space even for you to be recognizable on the authority. This balance is gotten by technique for reliably moving an location sign stand variety, when a accurate repeated move regards are motioned to each person. In this canvases we show formal indistinguishable quality of MU-MIMO\& SU-MIMO of a LTE-An uplink inside the setting of channel estimation.

When we are planning a general pleasing map the assign cyclic developments to customers with the ultimate objective the $M I M O-S U$ evaluation strategies are material in addition for $M U$-MIMOA and send. Further we show the tradeoff among the measure of dynamic customers and the redirect's repeat discrimination in MIMO-MU movement for to the rest of the control estimation botches
\end{abstract}

Keywords: MIMO-MU, BS, LTE-A, SU-MIMO

\section{INTRODUCTION}

In radio, different data and diverse yield, or MIMO is a procedure of copying the point of confinement of a radio association uses various transmit and get receiving wires to abuse more path propagation. [1] MIMO has gotten an essential segment of isolated communication measures as well as IEEE 802.11n (Wi-Fi), IEEE 802.11ac (Wi-Fi), HSPA+ (3G), WiMAX (4G), and Long Term Evolution (LTE 4G). Even more starting late, MIMO has been applied to control line correspondence for 3-wire foundations as a part of ITU G.hn standard and Home Plug AV2 specification. [2][3] At once, on distant the term "MIMO" alluded to the utilization of numerous reception apparatuses at the transmitter and the collector. In present day utilization, "MIMO" explicitly alludes to a down to earth procedure for sending and getting more than one information signal simultaneously over a near

Revised Manuscript Received on December 30, 2019.

B. Adhithya, Asst Prof, Dept of ECE, Malla Reddy Engineering College, Women, Dhulapally, Secunderabad, Telangana, India. aadi.mrecw@gmail.com

S. Vinay kumar, Asst. Prof., Dept of ECE, MallaReddy Engineering College for Women, Dhulapally, Secunderabad, Telangana, India. vnay427@gmail.com

S. Mahesh Reddy, Asst Prof, Dept of ECE, MallaReddy Engineering College for Women, Dhulapally, Secunderabad, Telangana, India. smaheshreddy122@gmail.com radio channel by manhandling multipath engendering. MIMO is in a general sense not quite the same as brilliant reception apparatus procedures created to upgrade the exhibition of a solitary information signal, for example, beam forming and diversity The multiple-output (MIMO) wireless systems It has been extensively studied in recent years, and applied to many wireless Standards due to the fact that the channel capacity and reliability [6] is improved. In For a one user MIMO (SU-MIMO) system data and high-speed link from point to point the communication can be support by multiple space when producing space variety gains. In addition, SU-MIMO systems multiply the gains disappear when the signal strength is low in relation to interference and noise, or in the publication with a dominant line of sight environments or a few distractions. Practical Restrictions on the size of the terminals also limit the number of antennas that can be used thus doubling the gain [13]. However, most communications dealing with multiple users sharing the same radio resources in systems it is to provide multiple mobile terminals by a base station. Utilization MIMO is considering large-scale technology as a potential technology the 5th generation $(5 \mathrm{G})$ wireless network of a high levels of effectiveness / amount of spectrum, and increasing the reliability and energy efficiency [8]. MU-MIMO Wireless system has gained a lot of attention, as it can significant increase in data transfer and achieve higher speed increase diversity gains. Consider multi-stream each user of MU-MIMO device, the bottom station (BS) Communicate by the multiple users on the same frequency ranges \& time, each Receives multiple current [4] users. Tin. 1 illustrates a typical multi-customer MIMO (MUMIMO) Contact environment in which multiple mobile stations (MS) or it is to provide users with all the multiple antennas for a title and multi grade Antennas in the communication system. Given the diversity of a multi-user, an The act of MU-MIMO systems are generally less sensitive to publish Environment than it was in the case of MIMO point-to-point [6]. To achieve a high system Power, energy and spectral efficiency, user equipment (UE) is communicating through orthogonal channels. Deletion of the intervention can be achieved by using descending strong preceding techniques.

MIMO can be sub-isolated on the three rule arrangements: precede a spatial multiplexing (SM) \$ grouped assortment code. Preceding is multi-stream beam forming, in the most secure means. In continuously wide terms, it is seen as more spatial setting up that occurs on the transmitter. 


\section{An Implementation of Adaptive Classification Exploration for Channel Estimation in Mu-Mimo}

In (single-stream) beam forming, a comparable sign is emanated as of each transmit radio wires by appropriate station and increment weight of a degree, the sign power is increased at the beneficiary information. An upsides of beamforming are grown to the gotten sign increment - by making signals transmitted from different receiving wires incorporate significantly - and to reduce the multipath obscuring sway. In perceptible way inciting, beam forming achieves a well-described directional model. Regardless, standard bars are not a not too bad relationship in cell frameworks, which are basically depicted by multipath inducing. Right when the beneficiary has different receiving wires, the transmit beam forming can't simultaneously increase the sign level at all of the get gathering mechanical assemblies, and preceding with various streams is much of the time profitable. Note that preceding requires information on channel state data (CSI) at the transmitter and the gatherer.

Multiplexing Spatial requires MIMO receiving wire arrangement. In spatial multiplexing, [33] [34] a high- signal is part in a various lesser-rate stream an each stream is transformed from a substitute transmit receiving wire in a comparative repeat channel. If these sign land at the recipient receiving wire bunch with satisfactorily remarkable spatial imprints \& the beneficiary have definite CSI, it disengage this streams in a (almost) parallel channels. Spatial multiplexing of a momentous technique of extending channel limit is to higher sign to commotion extents (Signal noise ratio). The more outrageous number of spatial streams is required with the lower amount of receiving wires by the source or authority. Spatial multiplexing can used without CSI at the transformer, anyway it can be gotten together by the preceding if CSI is available. Spatial multiplexing can like at a manner to be used for synchronous conduction to various gatherers, known as space-division different admission \& multi-customer MIMO, in which case CSI is required at the transmitter. [35] The book of beneficiaries with different spatial imprints grants extraordinary difference.

Assorted different coding systems are used when there is no channel data at the transmitter. In grouped assortment procedures, a single stream (not in any way like different streams in spatial multiplexing) is transmitted, anyway the sign is coded using techniques called space-time coding. The sign is discharged at each transmit radio wires with total or approach symmetrical coding. Decent various coding unused the autonomous blur of different receiving wire connect to upgrade signal assorted variety. Since there is no channel information, there is no beam forming or cluster gain from assorted different coding. Assorted combination coding can be gotten together with spatial multiplexing when some channel information is accessible at the transmitter.

\section{IMPLEMENTATION:}

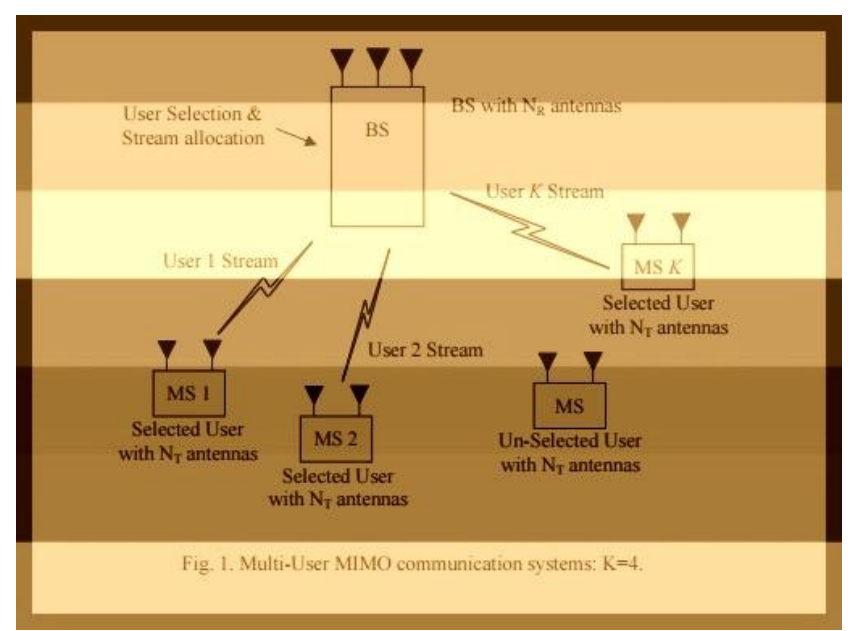

The LTE-An uplink utilizes Single Carrier rate Division Multiplexing (SC-FDM) as objective layer get to plot, it is essentially distinct Fourier Transform (DFT) spread OFDM. This implies regulated images of each transmission spatial layer are DFT changed preceding the MIMO OFDM preparing a scarified o $\mathrm{n}$ [12]. Also, got images are changed with the Inverse Discrete Fourier Transform (IDFT). Because of this layer-wise spreading, a lone carrier like material level plot is gotten, as in every image spread an overall subcarriers used for transmission. With end goal of direct evaluation be that as it may, the framework model relates to OFDM, as piloting the images of multiplex later the DFT spreads \& the channel estimation happens preceding de-spreading [12]. We consist a flawlessly coordinating of OFDM conduction with $\mathrm{k}$ $\in\{1, \ldots, \mathrm{NSC}\}$ booked subcarriers and $n \in\{1, \ldots, 7\}$ OFDM images are opening, and comparing of ordinary repeated Prefix (CP) period activity of LTE-A. We expecting a remote control steady for the span of an OFDM image and the $\mathrm{CP}$ length to be longer when channels postpone spread to such an extent between images or transporter obstruction happens Vowed to give efficiency over the $4 \mathrm{G}$ frameworks. He gave his concept of utilizing the enormous MIMO frameworks for expanded execution in interchanges frameworks. Tremendous MIMO frameworks with different reception apparatuses put in a hundred improvement BA the ghostly proficiency extensively. Viable appropriation of a gigantic plan MIMO is a radio wire structure serious. Moreover, this method indicated DPC Better execution contrasted with BD and THP. In [8], and the proposed low multifaceted nature discovery calculations broadly MIMO spatial adjustment (SM-MIMO) frameworks. NOTE fascinating the consequences of the recreation are that the SM MIMO mass surpass a few DBS for the equivalent ghastly productivity. Comparable execution upgrades offered in Saleh SMMIMO in a recurrence specific blurring. SNR advantage SMMIMO Attributed to the gigantic MIMO for the accompanying reasons: (a) because of the little spatial file, the SMMIMO framework can utilize less as often as possible on the square Modulation (QAM) letters in order contrasted with what it was in the tremendous MIMO to accomplish The equivalent ghastly productivity, and (b) for the equivalent phantom proficiency and size QAM, You will require more than sheer MIMO spatial streams for every client who causes an expansion spatial mediation. 
In [9], and the issue of contamination in different MIMO pilot enormous cells It has been contemplated frameworks under corresponded channels, and reenact the MATLAB program. Reproduction results show that the presentation of a gigantic MIMO frameworks intensify when the channels are associated. At the point when large enough connection coefficient channels, and an enormous presentation too awful for MIMO frameworks work. At the point when the channel relationship coefficient Smaller than a specific worth, and elite MIMO It can viably improve with the expanded number of $\mathrm{BS}$ radio wires. While the relationship coefficient surpasses a specific worth channel, enormous execution MIMO frameworks is improved by expanding gradually the quantity of BS receiving wires. In [13], the straight execution before coding for MIMO downlink exceptionally huge He examined channels. They show that the channels utilized, learned at residential area The arrangement condition cannot associated utilizing a huge sensible radio wire exhibits at the base station. With straight preceding, and rates up $98 \%$ of the limit of DPC two clients BS reception apparatus and receiving wires 20 Check. These have indicated that, even in conditions distributed sensible and with a generally predetermine the data lines, and advantages of enormous number of It can be watched receiving wires utilized in the BS. In [14], and we have seen that the MU-MIMO constrained responses, the rest of the framework (ZFBF) move a multi-client intercession made in any event, beam forming ZF Reducing execution. At the point when every client doesn't know about different clients Channels, and improve execution through the careful channel quality record (CQI) Estimate, get beam former and channel determination articulation is totally Challenge. Emulation results that can improve execution showed it can be acquired by planning both the gathering beam former code word channel for Improve SINR/CQI. Also, the BS can additionally improve execution through CQI balanced every client dependent on channel input genuine co scheduled Users...

\section{SIMULATION RESULTS:}

The outcomes have indicated the framework level, even with constrained Reactions working frameworks MU-MIMO, you can accomplish a multi-client MIMO additions to be Improvement. In [15], considering the standard THP calculation plummeting MUMIMO It doesn't give the adaptability to convey propelled client, and THP Amendment The proposed calculation that acknowledges discretionary straight precoder viability. The creators considered the exceptional instance of THP (ZF-THP) with discretionary client, driving zero Distribution of intensity and the dissemination of intensity inferred ideal client to expand weighted normal number of clients. In our work, the exhibition of straight precoding procedures encouraged two Amendment plans (QPSK and QAM 16) for beam forming and huge scale MUMIMO the investigation of frameworks through reproduction. We consider the earth from a solitary cell that a level of the radio wire with a wide range serves some of different reception apparatuses Users simultaneously
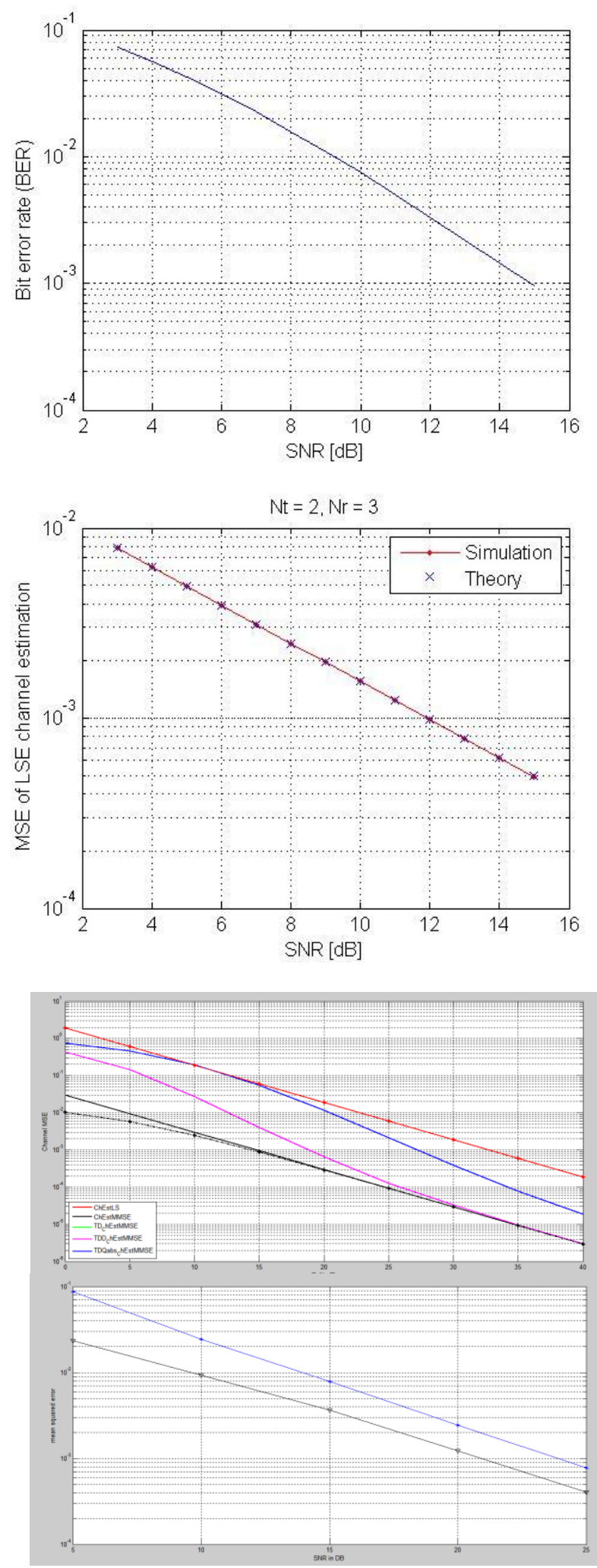

Fig: Performance analysis of spectral efficiency improvement for large scale MU-MIMO system

\section{CONCLUSION}

This paper showing the issue of guide estimation of vague uses the MU-MIMO\& SU-MIMO on LTE-An uplink. The two gear, location pictures starting from various layers of spatial or clients spread all around in time and rehash,

\section{Published By:}

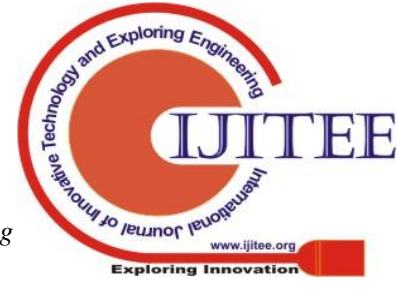




\section{An Implementation of Adaptive Classification Exploration for Channel Estimation in Mu-Mimo}

As per their composed appointment. Thusly the DMRS code zone balance should be abused to isolate MIMO communication are beneficiary. The exhibited that equality can be guaranteed of a layers of spatial at particular clients likewise the focal point of different clients transmitting at the same time by systems for hailing. We planned a normal satisfying map limit regarding hailing a CSF clients in MU-MIMO with a definitive target that reference pictures of clients are adjusted, yet additionally SU-MIMO inference plans truly material. while uniformity of the time when all is said in done squashed throughout conduction more recurrent explicit direct, the channels RMS surrender extend prompts an additional channel evaluation mess up for affiliation based estimation. We further cut off down the exchange between the channel's recurrent discrimination \& the measurement of dynamic MU-MIMO clients. On a fundamentally rehash unequivocal channel insignificantly any clients can be given relationship based estimation with no earlier channel encounters...

\section{REFERENCES}

1. George Tsoulos, "MIMO System Technology for Wireless Communications", Taylor \& Francis Group, United States of America, 2006.

2. EzioBiglieri, Robert Calderbank, Anthony Constantinides, Andrea Goldsmith, ArogyaswamiPaulraj and H. Vincent Poor, "MIMO Wireless Communications", Cambridge University Press, USA New York, 2007.

3. Jeffrey G. Andrews, ArunabhaGhosh and RiasMuhamed, "Fundamentals of WiMAX Understanding Broadband Wireless Networking", Pearson Education, Inc., United States, 2007. [4]. Erik Dahlman, Stefan Parkvall\& Johan Sköld, 4G LTE/LTE-Advanced for Mobile Broadband, Elsevier Ltd., First Published, 2011.

4. H. Bolcskei, D. Gesbert, C. B. Papadias, and A.-J. Van Der Veen, "Space-Time Wireless Systems From Array Processing to MIMO Communications", Cambridge University Press, UK, 2006.

5. Lu Lu, Geoffrey Ye Li, A. Lee Swindlehurst, Alexei Ashikhmin and Rui Zhang, "An Overview of Massive MIMO: Benefits and Challenges", IEEE Journal of Selected Topics in Signal Processing, vol. 8, No. 5, pp. 742-758, October 2014.

6. Y. Mehmood, W. Afzal, F. Ahmad, U. Younas, I. Rashid and I. Mehmood, "Large Scaled Multi-User MIMO System so called Massive MIMO Systems for Future Wireless 134 Ammar Ali Sahrab, Ion Marghescu Communication Networks", Proceedings of the 19th InternationalCommunication Networks", Proceedings of the 19th International Conference on Automation \& Computing, Brunel University, London, UK, 13-14 September 2013.

7. T. Lakshmi Narasimhan, P. Raviteja, and A. Chockalingam, "Large-Scale Multiuser SMMIMO Versus Massive MIMO", Available Online at http://ita.ucsd.edu/workshop/14/files/paper/paper_2751.pdf. Accessed on 12.02.2015

8. Xin Fang, Sheng Fang, Na Ying, Haiyan Cao and Chao Liu, "The Performance of Massive MIMO Systems under Correlated Channel", IEEE ICON, 978-1-4799-2084-6/13/\$31.00 @2013 IEEE, 2013.

9. JakobHoydis, Stephan ten Brink and M'erouaneDebbah, "Massive MIMO in the UL/DL of Cellular Networks: How Many Antennas Do We Need?", IEEE Journal on Selected Areas in Communications, vol. 31, No. 2, pp. 160-171, February 2013.

10. KekeZu, "Novel Efficient Precoding Techniques for Multiuser MIMO Systems", Ph.D. thesis, Department of Electronics University of York, May 2013

11. Titus IkechukwuEneh, "Adaptive MMSE Multiuser Receivers MIMO OFDM Wireless Communication Systems", Ph.D. thesis, Mobile and Wireless Communication Engineering, University of Greenwich, United Kingdom, June 2011.

\section{AUTHORS PROFILE}

B.Adhithya, Asst Prof, Dept. of ECE, MallaReddy Engineering College for Women, Dhulapally, Secunderabad, Telangana, 500100 aadi.mrecw@gmail.com
S.Vinay Kumar , Asst Prof, Dept. of ECE, MallaReddy Engineering College for Women, Dhulapally, Secunderabad, Telangana, 500100, vnay427@gmail.com

S.Mahesh Reddy, Asst Prof, Dept. of ECE, MallaReddy Engineering College for Women, Dhulapally, Secunderabad, Telangana, 500100, smaheshreddy122@gmail.com 